\title{
Corner Minus
}

National Cancer Institute

\section{Source}

National Cancer Institute. Corner Minus. NCI Thesaurus. Code C67054.

The average cell intensity for the antisense probe cells used in the grid alignment process. 